\title{
American College of Medical Genetics recommendations for the design and performance expectations for clinical genomic copy number microarrays intended for use in the postnatal setting for detection of constitutional abnormalities
}

\author{
Hutton M. Kearney, PhD ${ }^{I}$, Sarah T. South, PhD ${ }^{2}$, Daynna J. Wolff, PhD ${ }^{3}$, Allen Lamb, PhD ${ }^{4}$, Ada Hamosh, MD", \\ and Kathleen W. Rao, PhD ${ }^{6}, A$ Working Group of the American College of Medical Genetics
}

\begin{abstract}
Disclaimer: These ACMG Standards and Guidelines are developed primarily as an educational resource for clinical laboratory geneticists to help them provide quality clinical laboratory genetic services. Adherence to these standards and guidelines is voluntary and does not necessarily assure a successful medical outcome. These Standards and Guidelines should not be considered inclusive of all proper procedures and tests or exclusive of other procedures and tests that are reasonably directed to obtaining the same results. In determining the propriety of any specific procedure or test, the clinical laboratory geneticist should apply his or her own professional judgment to the specific circumstances presented by the individual patient or specimen. Clinical laboratory geneticists are encouraged to document in the patient's record the rationale for the use of a particular procedure or test, whether or not it is in conformance with these Standards and Guidelines. They also are advised to take notice of the date any particular standard or guidelines was adopted and to consider other relevant medical and scientific information that becomes available after that date. It also would be prudent to consider whether intellectual property interests may restrict the performance of certain tests and other procedures.
\end{abstract}

\begin{abstract}
Genomic copy number microarrays have significantly increased the diagnostic yield over a karyotype for clinically significant imbalances in individuals with developmental delay, intellectual disability, multiple congenital anomalies, and autism, and they are now accepted as a first tier diagnostic test for these indications. As it is not feasible to validate microarray technology that targets the entire genome in the same manner as an assay that targets a specific gene or syndromic region, a new paradigm of validation and regulation is needed to regulate this important diagnostic technology. We suggest that these microarray platforms be evaluated and manufacturers regulated for the ability to accurately measure copy number gains or losses in DNA (analytical validation) and that the subsequent interpretation of the findings and assignment of clinical significance be determined by medical professionals with appropriate training and certification. To this end, the American College of Medical Genetics, as the professional organization of board-certified clinical laboratory geneticists, herein
\end{abstract}

From the ${ }^{1}$ Fullerton Genetics Center, Mission Health System, Asheville, North Carolina; ${ }^{2}$ ARUP laboratories, Departments of Pediatrics and Pathology, University of Utah, Salt Lake City, Utah; ${ }^{3}$ Department of Pathology and Laboratory Medicine, Medical University of South Carolina, Charleston, South Carolina; ${ }^{4}$ Signature Genomics, Spokane, Washington; ${ }^{5}$ Department of Pediatrics and the McKusick-Nathans Institute of Genetic Medicine, Johns Hopkins University School of Medicine, Baltimore, Maryland; and ${ }^{6}$ Departments of Pediatrics and Pathology, School of Medicine, the University of North Carolina at Chapel Hill, Chapel Hill, North Carolina.

Hutton M. Kearney, PhD, Fullerton Genetics Center, Mission Health System, 267 McDowell St., Asheville, NC 28803. E-mail: Hutton.kearney@msj.org.

Disclosure: The authors declare no conflict of interest.

Published online ahead of print June 15, 2011.

DOI: $10.1097 /$ GIM.0b013e31822272ac outlines recommendations for the design and performance expectations for clinical genomic copy number microarrays and associated software intended for use in the postnatal setting for detection of constitutional abnormalities. Genet Med 2011:13(7):676-679.

Key Words: microarray, aCGH, CMA, guideline

Searching for a genetic etiology for intellectual disabilities, $S_{\text {developmental delays, and congenital anomalies is often }}$ difficult due to the high frequency of nonspecific features shared among numerous potential syndromes. Cytogenetic examination of banding patterns on metaphase chromosomes, the traditional karyotype analysis, has proven a useful diagnostic tool over the past 4 decades for the detection of these conditions. The goal of traditional cytogenetic analyses is to identify a specific genetic cause for the patient's symptoms by examining the genome in as much detail as possible, searching for an alteration of the typical chromosomal number or banding pattern. In many studies, the result might be easily anticipated (e.g. trisomy 21). However, in some cases, a novel or complex abnormality is discovered, even when the phenotype of the patient is highly suggestive of a different etiology. It is for this reason that, even when a newborn presents with features of a classic syndrome such as Down syndrome, the cytogeneticist does not simply analyze the region of interest in the case but rather examines the entire genome for unexpected significant imbalances (losses or gains) that may be present.

We now have new analytical tools, including genomic copy number microarrays (also known as cytogenetic microarrays or chromosomal microarrays), that detect genomic gains and losses with unprecedented resolution. As genomic microarrays have sig- 
nificantly increased the diagnostic yield over a karyotype for clinically significant imbalances in individuals with developmental delay, intellectual disability, multiple congenital anomalies, and autism, they are now accepted as a first tier diagnostic test for these indications. ${ }^{1-4}$ The introduction of genomic microarrays into clinical laboratories has presented unique validation and regulatory challenges. It is not feasible to validate a molecular technology that targets the entire genome in the same manner as an assay that targets a specific gene or syndromic region. Because this technology represents such a tremendous advance in diagnostic utility and patient care, we must accept these limitations and seek a new paradigm of validation and regulation.

We suggest that these microarray platforms be evaluated and manufacturers regulated for the ability to accurately measure copy number gains or losses in DNA (analytical validation) and that the subsequent interpretation of these findings and assignment of clinical significance be determined by medical professionals with appropriate training and certification. To this end, the American College of Medical Genetics, as the professional organization of board certified clinical laboratory geneticists, has outlined recommendations for the design and performance expectations for clinical genomic copy number microarrays and associated software intended for use in the postnatal setting for detection of constitutional abnormalities.

\section{RECOMMENDATIONS FOR MICROARRAY DESIGN, PROBE SELECTION, AND GENOMIC COVERAGE}

1. Probes should be placed throughout the genome at regular intervals, such that the microarray will detect genomic copy number variants (CNVs), both gains and losses, of $400 \mathrm{~kb}$ or larger, genome wide. This will allow for a broad genomic screen for novel imbalances and reliably detect all currently described syndromic microdeletions and microduplications mediated by segmental duplication architecture. Experience gained from the use of high-density genomic microarrays has shown that a significant proportion of patients with a diagnosis of a common deletion or duplication syndrome have atypical or complex rearrangements with clinical significance. High-density, whole-genome coverage allows for accurate delineation of breakpoints, which can allow for increased diagnostic certainty of the clinical consequence of the $\mathrm{CNV}$, particularly if the boundary regions contain relevant genes. The number of probes necessary to achieve this resolution will vary dependent on the platform used. The purpose is not to limit the resolution of clinical genomic microarrays; manufacturers are encouraged to exceed the minimum detection size of 400 $\mathrm{kb}$, when feasible through array design and performance.

2. It is desirable to have enrichment of probes targeting dosage-sensitive genes known to result in phenotypes consistent with common indications for a genomic screen (e.g., intellectual disability, developmental delays, autism, and congenital anomalies). Dosage-sensitive genes include those for which a deviation from the normal copy number state (through single copy gain or loss) has clinical implications for the patient. The purpose of this targeting is to maximize serendipitous detection of CNVs smaller than $400 \mathrm{~kb}$ in regions of known clinical relevance. The selection of targeted genes should be made in collaboration with medical professionals, preferably with expert consensus review, and consultation of the medical literature. It is important to clarify that regardless of probe enrichment, no microarray platform will detect all mutations associated with a given syndrome (i.e., will not detect very small deletions and point mutations). Therefore, the manufacturer and performing laboratories must clearly state that failure to detect a copy number alteration at any locus does not exclude the diagnosis of any of the disorders targeted on the microarray.

3. If the microarray platform is meant to replace alternative clinically validated technologies for the detection of gains or losses of particular regions of the genome (subtelomeric regions, syndromic microdeletions, etc.), the manufacturer and performing laboratory should ensure appropriate probe coverage in those genomic regions, such that the analytical sensitivity for the typical imbalances seen in the patient population meets or exceeds that achieved with the alternative technology.

4. Microarrays that also assess genotype at common sites of single-nucleotide polymorphisms (SNPs) can determine copy number state and reveal regions of homozygosity, which may indicate uniparental disomy and/or direct attention to potential candidate genes responsible for recessive conditions. SNP allele ratios can also provide supportive evidence for copy number state and mosaic conditions. Therefore, inclusion of SNP probes is a desirable feature of a clinical genomic microarray, provided that there is appropriate genomic coverage and performance for detection of CNVs, as outlined in this document. SNP analysis is not a requirement at present for clinically appropriate genomic microarrays, as the primary goal of these analyses is to reliably detect copy number alterations.

5. Probes that target repetitive sequences and/or show spurious calls as gains or loss that do not represent true copy number variation in the sample should be avoided (or permanently suppressed from analysis). See section on analytical specificity. It is recognized and expected that this will result in gaps in genome-wide coverage, particularly in regions with many repetitive elements. The manufacturer should clearly state the limitations of the design by outlining which regions of the genome are covered at regular intervals and which regions have no probe coverage.

6. Before a design is finalized, manufacturers should challenge the microarray with a comprehensive set of abnormal samples that in combination survey all regions of the genome represented on the microarray. Those probes that fail to demonstrate appropriate and reliable copy number response should be excluded (or permanently suppressed from analysis). The source of DNA for this purpose may be any well-characterized cell line or control specimen. Artificial (spiked) controls may also be considered, provided there is reasonable assurance that the conditions mimic the relative copy number for biologically relevant gains and losses. If only a deletion control is available, it should be ensured that probes in the interval show appropriate dynamic range for detection of single copy gains.

\section{RECOMMENDATIONS FOR MANUFACTURER'S PREMARKET ANALYTICAL VALIDATION}

The goal of the manufacturer's premarket analytical validation process should be (1) to identify the parameters specific to their platform (number of consecutive probes, $\log _{2}$ ratio, SNP allele ratios, quality control metrics, etc.) and to specify an appropriate software algorithm necessary to achieve $\sim 99 \%$ 
detection of $\mathrm{CNVs}>400 \mathrm{~kb}$, (2) to establish a recommended metric of confidence that allows for $<1 \%$ false-positive calls of any size, and (3) ideally, to provide confidence intervals at copy number transition boundaries, and therefore accurate assessment of genomic content within the detected CNV. Because these considerations are dependent on probe density and performance and software algorithms, the following recommendations apply both to microarray and software manufacturers.

\section{Analytical sensitivity}

7. Manufacturers should detail the assay performance, quality parameters, and software algorithm required to achieve a target $99 \%$ analytical sensitivity of CNVs $>400 \mathrm{~kb}$ in the regions covered by the platform. The assessment of the analytical sensitivity may be first made by testing a sufficiently large number (200-300 or more) of well-characterized cases to establish a $\sim 99 \%$ analytical sensitivity for CNVs at least $400 \mathrm{~kb}$ in size, with a lower limit of the $95 \%$ confidence interval $>98 \%$. Ideally, these cases should contain unique CNVs with defined copy number alterations throughout the genome, with the majority representing CNVs $<1 \mathrm{Mb}$ in size. These samples should be derived from the same tissue source(s) using the DNA extraction procedures recommended in the manufacturer's protocol. A combination of these actual experimental challenges with further in silico data modeling approaches (simulation of additional abnormalities) should provide reasonable confidence that $>99 \%$ of all copy number alterations $>400$ $\mathrm{kb}$ will be detected with the microarray platform.

\section{Analytical specificity}

8. It is not feasible to calculate specificity in the usual way, as even phenotypically normal individuals are likely to have one or more CNVs present in the genome. Rather, one can determine the false-positive rate per CNV call. This can be achieved using the same samples from the analytical sensitivity study. Manufacturers should detail the parameters specific to their platform (number of consecutive probes, $\log _{2}$ ratio, SNP allele ratios, quality control metrics, etc) that are necessary to conclude that a copy number call represents true copy number variation. Any CNV call in the sample set meeting these parameters, regardless of pathogenicity, should be confirmed by an independent methodology with a target false-positive rate $<1 \%$. The $95 \%$ confidence interval around the falsepositive rate should also be determined and will be based on the number of CNVs identified in this study. It is expected that the parameters (e.g., number of consecutive probes) necessary to achieve this low false-positive rate will likely be more stringent for gains versus losses, as the expected probe ratios for single copy gains are more similar to the normal copy number state.

a. At the manufacturer's discretion, CNVs $<400 \mathrm{~kb}$ may also be called as long as this does not increase the false-positive rate. With proper technical performance and analytical validation, the performing laboratory should not be required to confirm a $\mathrm{CNV}$, regardless of size, called with the manufacturer's recommended parameters.

b. In addition to the overall confidence with which a $\mathrm{CNV}$ is called, it is recommended that confidence measures also be assigned to probes/regions at the boundaries of a CNV to define the breakpoints as- signed and provide appropriate minimum/maximum CNV intervals.

c. Given that it is desirable to maximize detection of aberrations below the $400 \mathrm{~kb}$ threshold that result in deletion or loss of function of clinically important genes and of aberrations in mosaic form (both of which may not generate a robust copy number call), it is acceptable and appropriate for the software to highlight certain calls that do not meet the stringent confidence parameters. At the discretion of the performing laboratory, such low confidence calls may be flagged for review and correlation with the patient's clinical indication but should be confirmed by an independent methodology if reported.

\section{CONSIDERATIONS REGARDING ANALYSIS AND INTERPRETATION OF GENOMIC MICROARRAY FINDINGS}

9. For the same reasons that we do not recommend microarrays targeted only to well-characterized syndromic regions, we strongly discourage manufacturers' use of in silico data filters that blind the performing laboratory to all data outside the targeted regions. These filters have been suggested to allow for interrogation of only those regions of the genome that are well characterized in the medical literature. We believe that blinding the interpreting geneticist to parts of the genome can lead to oversimplification and misinterpretation of complex and atypical abnormalities and may in turn result in missed or incorrect diagnoses. For this reason, even an array designed to target known pathogenic regions should be analyzed and interpreted using the same parameters outlined in this document, and in the context of a whole-genome analysis, rather than through the use of overly simplified reporting software.

10. Performing laboratories may wish to establish size restrictions for their CNV calls, and these parameters may be different for deletions versus duplications or syndromic regions versus uncharacterized regions of the genome. Manufacturers are encouraged to provide software that enables the performing laboratory to customize their analysis with such tools and additionally allows them to custom annotate regions on the microarray according to the growing evidence base. The selection and application of analysis and interpretation tools should be at the discretion of the (appropriately board certified) interpreting geneticist.

11. It is recognized that with increased genomic coverage, there will be both increased detection of pathogenic $\mathrm{CNVs}$ and increased detection of CNVs of uncertain clinical significance and CNVs that are likely benign. The interpretation and appropriate clinical reporting of these findings are complex, and are the practice of medicine. ${ }^{5}$ We recommend that the reporting of clinical genomic microarray findings be performed by individuals with appropriate professional training and certification (American Board of Medical Genetics-certified clinical cytogeneticists, American Board of Medical Genetics-certified clinical molecular geneticists, or American Board of Medical Genetics/American Board of Pathology-certified molecular genetic pathologists). Because many of the abnormalities identified by genomic microarrays result from cytogenetic rearrangements, it is often necessary to characterize the mechanism responsible for the imbalance for appropriate genetic counseling. If the microarray assay is performed in 
a molecular genetics laboratory, this laboratory should establish a close partnership with a cytogenetics laboratory, such that abnormal results have cytogenetic characterization and interpretation, as appropriate, before reporting. Family members should also be provided appropriate follow-up testing. With these considerations in mind, the ideal clinical laboratory setting for genomic copy number microarray analysis is one with both molecular and cytogenetic expertise.

\section{REFERENCES}

1. Manning M, Hudgins L; Professional Practice and Guidelines Committee. Array-based technology and recommendations for utilization in medical ge- netics practice for detection of chromosomal abnormalities. Genet Med 2010; 12:742-745.

2. Miller DT, Adam MP, Aradhya S, et al Consensus statement: chromosomal microarray is a first-tier clinical diagnostic test for individuals with developmental disabilities or congenital anomalies. Am J Hum Genet 2010;86:749-764.

3. Stavropoulos J. CCMG guidelines for genomic microarray testing. CCMG Cytogenetics Committee. Available at: http://www.ccmg-ccgm.org/pdf/policy/ 2010/(7)\%20CCMG_PractGuide_CYTO_Microarray_13July10.pdf. Accessed June 9, 2011.

4. Farndon P, Westwood J. Supporting genetic testing in the NHS - second report of the UKGTN. South East London, England: UKGTN, 2010.

5. Kearney HM, Thorland EC, Brown KK, Quintero-Rivera F, South ST; A Working Group of the American College of Medical Genetics (ACMG) Laboratory Quality Assurance Committee. American College of Medical Genetics standards and guidelines for interpretation and reporting of postnatal constitutional copy number variants. Genet Med 2011;13:680-685. 